\title{
L'évaluation de la qualité des espaces publics : un outil d'aide à la décision
}

\section{Assessing the quality of public spaces: A proposed decision support tool \\ La evaluación de la calidad de espacios públicos: Un útil de ayuda a la toma de decisión}

\section{Lydia Hadji}

Volume 57, numéro 160, avril 2013

Version originale soumise en juillet 2012. Version révisée reçue en mars 2013.

URI : https://id.erudit.org/iderudit/1017803ar

DOI : https://doi.org/10.7202/1017803ar

Aller au sommaire du numéro

Éditeur(s)

Département de géographie de l’Université Laval

ISSN

0007-9766 (imprimé)

1708-8968 (numérique)

Découvrir la revue

Citer cet article

Hadji, L. (2013). L'évaluation de la qualité des espaces publics : un outil d'aide à la décision. Cahiers de géographie du Québec, 57(160), 25-40.

https://doi.org/10.7202/1017803ar
Résumé de l'article

Dans un esprit de développement durable, les grandes villes s'efforcent d'atteindre de nouveaux objectifs qui mettent en question l'aménagement de l'espace public. La recherche d'un outil d'aide à la décision pour l'évaluation de la qualité des espaces publics devient une nécessité primordiale. Face à ce défi, il faut aborder l'espace public en tant que système compris comme un organisme holistique. Cette approche nous a permis d'établir une méthode d'évaluation de la qualité de l'espace public basée sur une série de critères et d'indicateurs d'évaluation scientifiquement valides et en mesure d'appuyer les décideurs dans leurs choix, cela, dans une perspective de développement durable. La méthode pourrait s'appliquer, en outre, dans les pays en développement tels que l'Algérie qui ne dispose pas d'outils d'évaluation de la qualité des espaces publics afin d'assurer une qualité de vie urbaine. 


\title{
L'évaluation de la qualité des espaces publics: un outil d'aide à la décision
}

\author{
Assessing the quality of public spaces: \\ A proposed decision support tool \\ La evaluación de la calidad de espacios \\ públicos: Un útil de ayuda a la toma de \\ decisión
}

\author{
Lydia HADJI \\ Laboratoire Ville Urbanisme et Développement \\ Durable, École Polytechnique et d'Urbanisme \\ Algérie \\ Hadjilydia@gmail.com
}

\section{Résumé}

Dans un esprit de développement durable, les grandes villes s'efforcent d'atteindre de nouveaux objectifs qui mettent en question l'aménagement de l'espace public. La recherche d'un outil d'aide à la décision pour l'évaluation de la qualité des espaces publics devient une nécessité primordiale. Face à ce défi, il faut aborder l'espace public en tant que système compris comme un organisme holistique. Cette approche nous a permis d'établir une méthode d'évaluation de la qualité de l'espace public basée sur une série de critères et d'indicateurs d'évaluation scientifiquement valides et en mesure d'appuyer les décideurs dans leurs choix, cela, dans une perspective de développement durable. La méthode pourrait s'appliquer, en outre, dans les pays en développement tels que l'Algérie qui ne dispose pas d'outils d'évaluation de la qualité des espaces publics afin d'assurer une qualité de vie urbaine.

\section{Mots-clés}

Évaluation, outil d'aide à la décision, qualité de l'espace public, système.

\begin{abstract}
In the interests of sustainable development, cities today need to establish new goals that challenge previous ideas about how public spaces should be designed. In this changed context, there is a vital need for appropriate decision support tools to assess the quality of public spaces. One way to address this challenge is through a holistic approach where public spaces are understood as whole systems. We adopted this approach and developed a method for decision-makers to apply in assessing the quality of public spaces from a sustainable development perspective, using a scientifically valid series of criteria and evaluation indicators. Notably, the method described could be of particular use in developing countries such as Algeria which, although wishing to ensure their citizens have good quality urban life, do not currently have the decision support tools they need to assess the quality of public spaces.
\end{abstract}

\section{Keywords}

Assessment, decision support tools, quality of public spaces, systems.

\section{Resumen}

Intrínsecamente al pensamiento de desarrollo sostenido, las grandes ciudades se esfuerzan de alcanzar nuevos objetivos que refutan el planeamiento del espacio público. Constituye una necesidad primordial la búsqueda de un instrumento de ayuda a tomar decisiones para poder 
evaluar la calidad de los espacios públicos. Frente a ese desafío, se debe abordar el espacio público en tanto que sistema entendido como un organismo global. Este enfoque nos permite plantear un método de evaluación de la calidad del espacio público, el que está basado en criterios e indicadores de evaluación científicamente válidos. Serán capaces de apoyar a los que toman decisiones tanto en sus opciones que en la perspectiva de desarrollo sostenido. Además, este método podría aplicarse a otros países en proceso de desarrollo como Algeria, aquellos que no disponen de instrumentos de evaluación de la calidad de los espacios públicos que aseguren una vida urbana mejor.

\section{Palabras claves}

Evaluación, útil de ayuda a la toma de decisión, calidad del espacio público, sistema.

\section{Introduction}

Dans un esprit de développement durable, les grandes villes s'efforcent d'atteindre de nouveaux objectifs qui mettent en question l'aménagement de l'espace public et les usages qu'on en fait. La recherche d'une qualité de l'espace public devient une nécessité primordiale. Les approches généralement utilisées portent un regard singulier sur l'espace public et sa qualité, par exemple, l'approche de sociologie urbaine où l'espace public est considéré comme un événement où des rituels d'interaction sont visibles et observables (Sennett, 1979), ou bien celle de la sociologie de la vie quotidienne, qui considère l'espace public comme un lieu d'action et des signes à partir du modèle de la théâtralité (Goffmann, 1973). L’approche de philosophie politique définit l'espace public comme un espace de débats, de conversations et de controverses dans une ambiance démocratique, une sphère de publicité au sens de Kant, c'est-à-dire où l'on peut appliquer «l'usage public de la raison », parler librement sans contrainte, défendre ses opinions et formuler ses réflexions dans un lieu où s'entremêlent anonymat et diversité de tout genre. Cette approche est fondée sur les théories de philosophie politique des années 1960 d'Hannah Arendt et de Jürgen Habermas, où l'espace public représente une scène d'apparition publique et où la diversité des opinions s'exprime (Devillard et Jannière, 1997). Par ailleurs, l'approche spatiale et urbaine définit l'espace public comme un lieu d'urbanité par excellence, générant des formes urbaines. L'approche paysagère et perceptuelle vise la dimension et la qualité visuelle et plastique de l'espace (Lynch, 1969, [1960]) ; Cullen, 1961). Ici, l'espace public est un espace visuel, olfactif, auditif, tactile et kinesthésique (Hall, 1966).

Chacune de ces approches aborde un seul aspect de l'espace public sans une vision holistique, ce qui ne permet pas d'évaluer la qualité des espaces publics dans un contexte de durabilité et dans un objectif d'aide à la décision. Face à ce défi, il faut aborder l'espace public en tant que système compris comme un organisme holistique (Berezowska - Azzag, 2012), avec ses sous- systèmes (dimensions) et des composants (critères de qualité).

Notre objectif, dans cet article, est de présenter une méthode d'évaluation de la qualité de l'espace public qui puisse servir d'outil d'aide à la décision. Cette méthode pourrait s'appliquer, en outre, dans les pays en voie de développement tels que l'Algérie qui ne dispose pas d'outil d'évaluation de la qualité des espaces publics afin d'assurer une qualité de vie urbaine. 


\section{Démarche de construction du modèle}

L'application du développement durable à la ville vise à améliorer la qualité de vie, à apporter le bien-être à la population locale pour une ville prospère à long terme. Cette qualité de vie sera obtenue grâce, entre autres, à l'amélioration de la qualité de l'espace public. Ce dernier est au cœur de l'interaction des trois sous-systèmes - économique, environnemental et social - qui forment le système urbain. Il est un champ d'articulation des objectifs du développement durable urbain (figure 1).

\section{Figure 1 L'espace public au cœur de l'interaction des trois domaines du système}

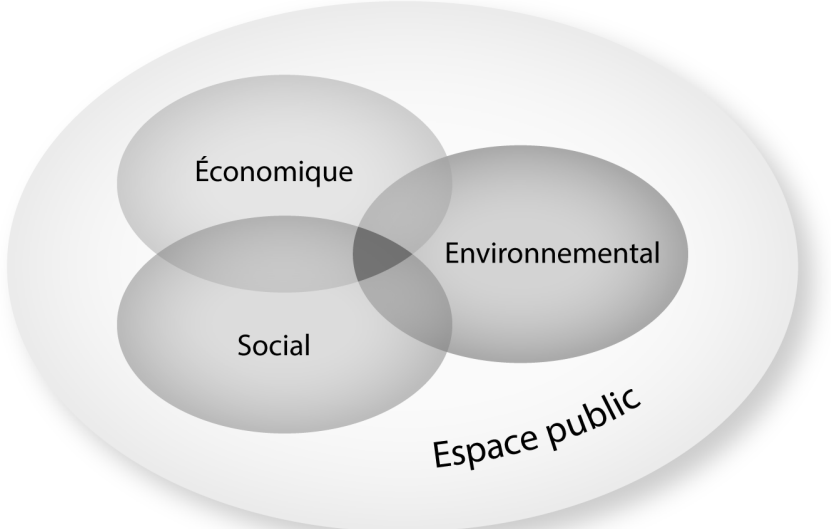

Pour la construction du modèle, la démarche proposée consiste à traiter l'espace public comme un système évolutif avec des sous-systèmes (dimensions) qui sont en lien entre eux et cumulant des critères. Ces derniers sont issus des différentes approches. Pour cela, on se rapproche de la position défendue par Sen (1993), dans le champ de l'évaluation des dimensions du bien-être, adaptée aux contextes particuliers de

l'étude et affinée par la confrontation de plusieurs sources ou avis pour l'établissement des critères d'évaluation du bien-être. À cet effet, on se base sur:

- un état des savoirs des différentes approches abordant l'espace public,

- un questionnaire en ligne transmis à des professionnels de l'urbain (concepteurs, décideurs et gestionnaires) ${ }^{1}$,

- $\quad$ un questionnaire in situ qui vise les usagers des espaces publics ${ }^{2}$,

- des référentiels existants ${ }^{3}$.

1 Le questionnaire ouvert cible les professionnels de l'urbain pour identifier les critères de qualité des espaces publics. Il est transmis par Internet à 30 chercheurs universitaires de l'École Polytechnique d'Architecture et d'Urbanisme qui sont, pour la plupart, des architectes de formation. Il est aussi transmis à 10 décideurs et gestionnaires des collectivités locales et à 10 architectes praticiens.

2 Ce questionnaire a ciblé 100 usagers de certains espaces publics algérois.

3 Citons les travaux de recherche de Catherine Charlot-Valdieu et Philippe Outrequin qui ont présenté, dans leur ouvrage (2009), un référentiel qui devait être discuté avec les acteurs urbains (l'aménageur et le maître d'ouvrage) afin de définir des objectifs concrets permettant de donner une cohérence d'ensemble au projet. Ce référentiel comporte sept thèmes (dont les mots-clés forment le nom de ce référentiel, MEFISTO) : gestion de la Mobilité, interface entre les Espaces privés et publics, gestion des Flux, Intégration du projet dans la ville, Suivi des chantiers, Traitement des espaces publics, Occupation de l'espace. Les référentiels locaux concernent le Grand projet urbain (GPU), le Plan d'aménagement côtier de l'Algérois (PACA), le Plan directeur d'aménagement et d'urbanisme (PDAU) d'Alger «projet d'embellissement, de déplacement et de développement maîtrisé à l'horizon 2020 » et le Plan d'aménagement et de développement de la baie d'Alger. 
Sur la base de ces approches plurielles, nous avons construit le modèle «espace public » avec ses critères de qualité, issus des différentes entrées et se rapportant aux trois domaines du développement durable (économique, social et environnemental) (figure 2).

\section{Figure 2 Les critères de qualité du modèle « espace public »}

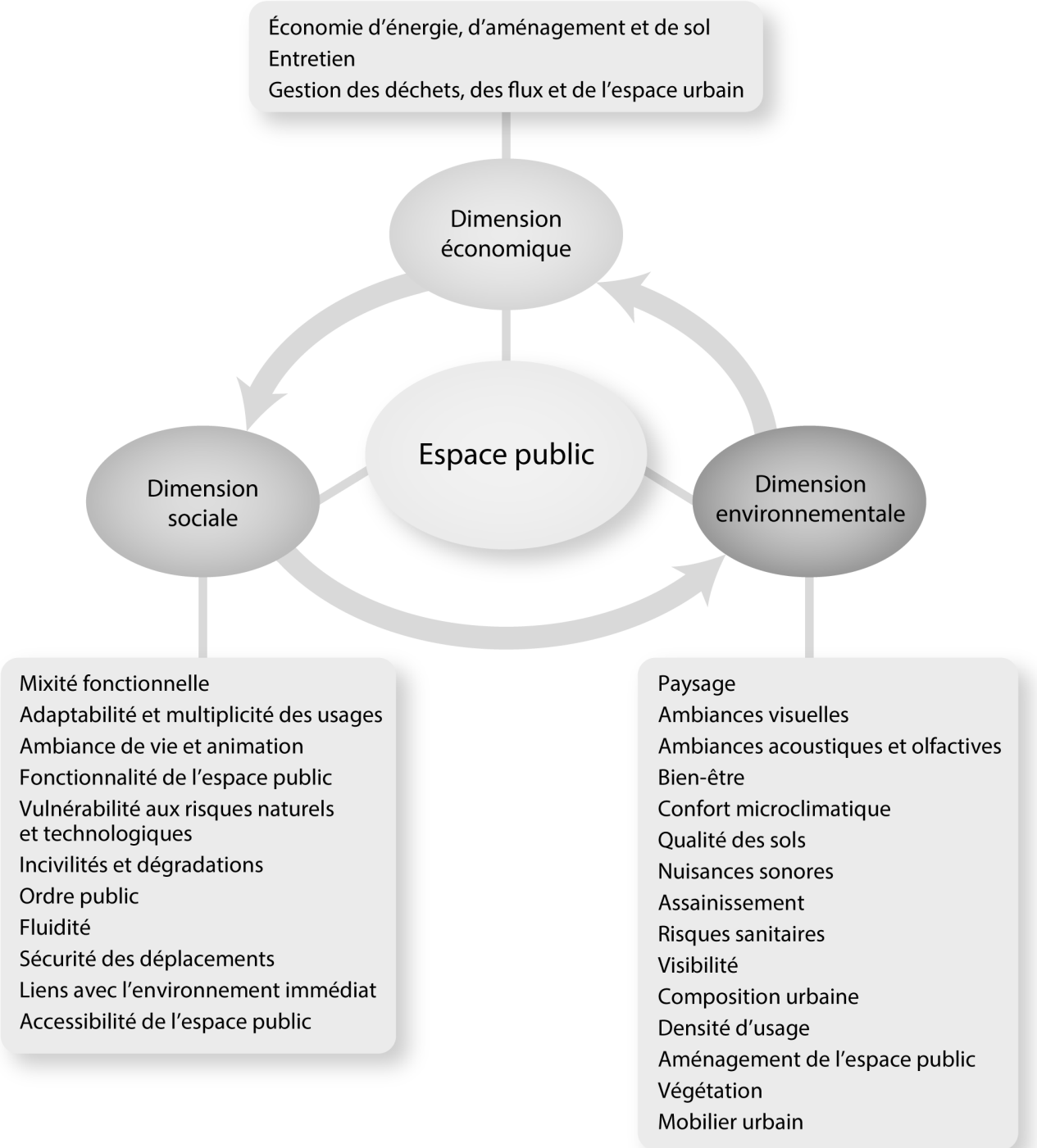




\section{Méthode d'évaluation de la qualité des espaces publics comme outil d'aide à la décision}

L'aide à la décision est définie, selon Roy et Bouyssou (1993), comme étant l'activité de celui qui, prenant appui sur des modèles clairement explicités mais non nécessairement complètement formalisés, aide à obtenir des éléments de réponse aux questions que se pose un intervenant dans un processus de décision, éléments concourant à éclairer la décision.

Pour la construction de la méthode d'évaluation de la qualité des espaces publics, nous avons regroupé les critères détaillés issus des différentes entrées afin de sélectionner des critères synthétiques qui faciliteront la mise en œuvre de cette méthode par les praticiens locaux. À cet effet, nous aurons 10 critères synthétiques d'évaluation de la qualité des espaces publics (figure 3) et une série d'indicateurs d'évaluation.

Figure 3 Le système des critères synthétiques d'évaluation de la qualité de l'espace public

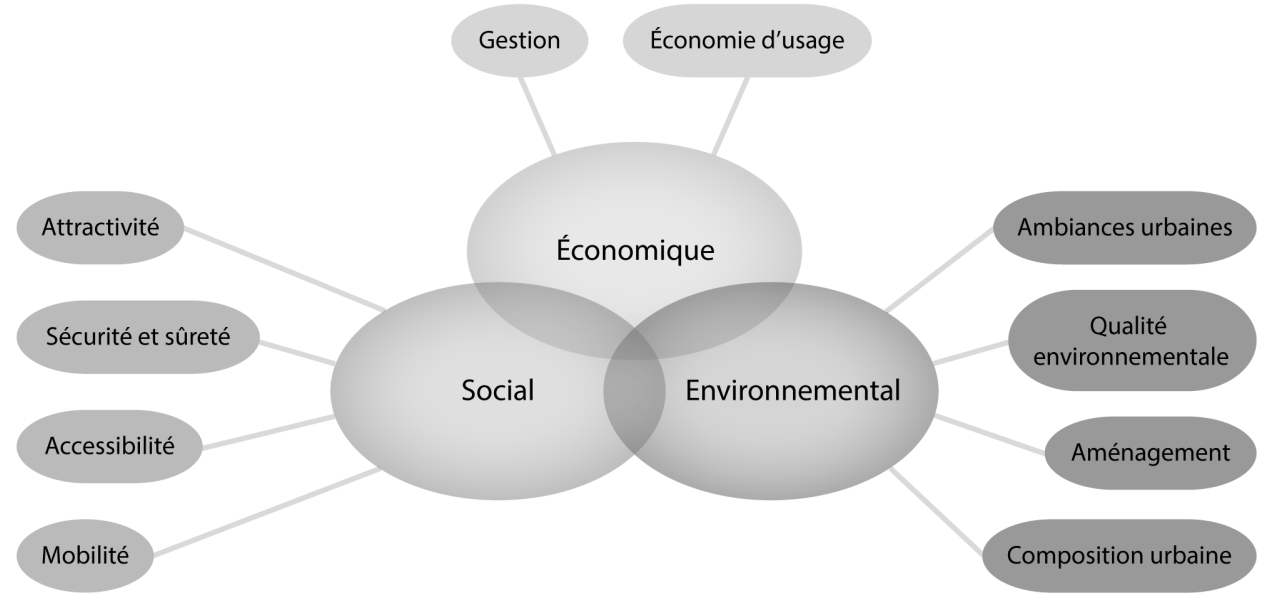

\section{Le tableau de bord}

Le tableau de bord de la méthode est une grille générale composée de 10 critères synthétiques définis précédemment et de 75 indicateurs d'évaluation de la qualité des espaces publics ${ }^{4}$, scientifiquement valides et en mesure d'appuyer les décideurs dans leurs choix, cela, dans une perspective de développement durable (Hadji, en cours) (tableau 1).

4 Les 75 indicateurs d'évaluation de la qualité des espaces publics sont le résultat d'un travail de recherche élaboré dans le cadre de ma thèse de doctorat (Hadji, en cours). 


\section{Tableau 1 Extrait du tableau de bord d'évaluation de la qualité des espaces publics}

\begin{tabular}{ll}
\hline $\begin{array}{l}\text { Critères synthétiques } \\
\text { issus de différentes } \\
\text { entrées d'investigation }\end{array}$ & $\begin{array}{l}\text { Indicateurs d'évaluation } \\
\text { de la qualité de l'espace public }\end{array}$ \\
\hline Mobilité & $\begin{array}{l}\text { Offre de places de stationnement } \\
\text { Offre d'espaces « deux-roues » (modes doux) } \\
\text { Offre d'espaces d'accueil de flux piétons (parvis) } \\
\text { Largeur des voies pour les espaces véhicules } \\
\text { Existence de cheminements adaptés et sans obstacles } \\
\text { Existence d'aménagements spécifiques pour les personnes } \\
\text { à mobilité réduite (PMR) } \\
\text { Existence de panneaux de signalisation et des éléments de repère } \\
\text { Offre de places de stationnement temporaire (approvisionnement, } \\
\text { urgence...) }\end{array}$ \\
\hline
\end{tabular}

\section{Procédure d'évaluation}

L'évaluation se fait en trois temps : la pondération, la notation et l'agrégation (figure 4).

\section{Étape de pondération (consultation d'acteurs)}

Diverses méthodes de pondération des critères existent et leurs résultats peuvent influencer le résultat final de l'analyse. Il y a les méthodes d'évaluation directe par le classement simple, comme la méthode d'attribution des scores (fixed point scoring) ou la méthode des comparaisons successives (Churchman et Ackoff, 1954), ou encore les méthodes indirectes comme la comparaison par paire (paired comparison) (méthode AHP [Saaty, 1977] et méthode MACBETH [Bana e Costa et al., 2005]).

Dans notre méthode d'évaluation, nous allons en premier lieu pondérer les critères selon les avis des usagers-habitants et des professionnels de l'urbain (concepteurs, décideurs et gestionnaires) par des questionnaires (in situ et en ligne) et des entretiens ciblés ${ }^{5}$ (Hadji, en cours). En second lieu, nous allons pondérer les indicateurs selon les avis des experts chargés d'évaluation, et ce, en nous basant sur la méthode d'attribution des scores (fixed point scoring), qui est la répartition d'une somme de points sur l'ensemble des critères ou des indicateurs en utilisant un arbre de pondération (Boulanger, 2004). Dans ce cas, les scores correspondent aux poids. Le choix de cette pondération repose sur sa simplicité et sa facilité d'utilisation sans le recours au logiciel.

La somme des scores des critères pondérés et la somme des scores des indicateurs pondérés relatifs à chaque critère doivent être égales à 10. Le score cumulé de l'indicateur $(S c)$ est exprimé par les coefficients de pondération cumulés sous forme d'un produit entre les scores de l'indicateur $\left(\mathrm{S}_{\text {ind }}\right)$ et du critère pondéré $(\mathrm{C})$. La somme des scores cumulés doit être égale à 100.

5 Un premier questionnaire in situ a ciblé 100 usagers - habitants ( 50 hommes et 50 femmes) aux abords de certaines stations de métro et de tramway. Un deuxième questionnaire en ligne a été transmis à des professionnels de l'urbain (chercheurs et praticiens). Nous avons effectué aussi des entretiens avec les décideurs et les gestionnaires de l'Entreprise du Métro d'Alger (EMA). 
Cette méthode de pondération est plus cohérente dans les comparaisons entre les critères. Elle établit les liens logiques : lien horizontal des cohérences des scores entre les indicateurs, lien vertical des cohérences des scores entre les indicateurs et les critères (Boulanger, 2004). Notons que l'attribution d'un score plus important à un critère ou à un indicateur réduit l'importance relative d'un autre critère ou indicateur (Cherqui, 2005).

\section{Étape de notation des indicateurs (par les experts-évaluateurs)}

Notons que l'exercice de notation est, par essence, un exercice subjectif, mais qui se base nécessairement sur l'observation, l'étude des documents et des échanges avec les professionnels. On peut apprécier le respect des différents indicateurs d'un point de vue synthétique en positionnant le projet vis-à-vis d'un barème (échelle de notation) compris entre 1 et 3 , choisi en fonction du contexte d'action ou d'évaluation, ce qui facilite aux évaluateurs l'utilisation de la méthode. On attribue 1 à la faible performance, 2 au seuil de performance et 3 au niveau très performant. Pour effectuer une évaluation selon le barème fixé au préalable, cette valeur mesurée doit être comparée à une valeur de référence, à partir d'un référentiel issu des différentes recherches bibliographiques. Comme exemple, nous allons exposer le référentiel du critère «mobilité» avec deux de ses quatre indicateurs qui nous semblent les plus représentatifs (tableau 2 ).

La valeur de l'indicateur (V) est une valeur pondérée et correspond à la multiplication du score cumulé $(S c)$ par la note de l'indicateur $(\mathrm{N})$.

\section{Étape d'agrégation}

L’agrégation introduit la vision synthétique de la qualité des espaces publics, qui favorise la prise de décision. À cet effet, on privilégie une approche pleinement agrégative à deux niveaux. À chaque niveau d'agrégation, l'ensemble des données est inclus dans une formule mathématique, afin d'obtenir un nombre de valeurs réduit à la fin de ce processus d'agrégation et de faciliter l'usage par les décideurs.

\section{1er niveau d'agrégation}

Pour que les indicateurs puissent servir aux processus de décision politique, leur nombre doit être réduit et la grille ou le système d'indicateurs simplifié. Nous rattachons en premier lieu les critères aux indicateurs synthétiques; nous aurons donc le même nombre de critères et d'indicateurs synthétiques. En suivant la Weight Sum Method (WSM), qui est le modèle des sommes pondérées, nous allons agréger la valeur des indicateurs synthétiques (VI.S $)$ relative à chaque critère. Celle-ci sera définie simplement comme la somme des valeurs pondérées des indicateurs de chaque critère.

VI. $S_{x}=\sum_{i=1}^{n} \mathrm{~V}_{\mathrm{X} . \mathrm{n}}=\sum_{i=1}^{n}\left(\mathrm{Sc}_{\mathrm{x} . \mathrm{n}} \times \mathrm{N}_{\mathrm{X} . \mathrm{n}}\right)$

$V_{x . n}=$ La valeur pondérée de l'indicateur.

$S c_{x . n}=$ Le score cumulé accordé à l'indicateur.

$N_{x . n}=$ La note obtenue par l'indicateur $i$. 


\section{Tableau 2 Le référentiel du critère « Mobilité ॥}

\begin{tabular}{|c|c|c|}
\hline \multicolumn{3}{|l|}{ Critère: Mobilité } \\
\hline Indicateur 1 & Indicateur 2 & $\mathrm{~T}$ \\
\hline Offre de places de stationnement & Offre d'espaces « deux roues" (mode doux) & $\uparrow$ \\
\hline $\begin{array}{l}\text { Définition: } \\
\text { Physiquement délimitée par rapport aux } \\
\text { espaces piétonniers et aux autres espaces: } \\
\text { lincolns, stationnement longitudinal sur } \\
\text { chaussée. } \\
\text { Orientations d'aménagement: } \\
\text { - La surveillance et le contrôle du respect de } \\
\text { la réglementation du stationnement devront } \\
\text { être renforcés. } \\
\text { - Le principe général préconisé est } \\
\text { d'aménager le stationnement le long des } \\
\text { chaussées en files longitudinales. Le } \\
\text { stationnement longitudinal pourra } \\
\text { s'envisager selon deux configurations: } \\
\text { - une configuration sur chaussée, le long } \\
\text { des trottoirs, matérialisée par un } \\
\text { marquage au sol; } \\
\text { - une configuration de type «lincoln»: } \\
\text { elle permet de bénéficier de sur-largeurs } \\
\text { de trottoirs au droit des passages piétons } \\
\text { notamment, assurant ainsi une meilleure } \\
\text { sécurité, mais permettant aussi la } \\
\text { continuité du fil d'eau et la régularité de } \\
\text { son tracé. } \\
\text { - } 1 \text { place sur } 50 \text { pour les handicapés, réservée } \\
\text { et accessible. } \\
\text { - La largeur de la place de stationnement est } \\
\text { de } 3,3 \text { m. } \\
\text { longitudinal: } \\
\text { - } 5 \text { m en file courante; } \\
\text { - } 5,5 \text { à } 6 \text { m en place unique entre } \\
\text { obstacles. } \\
\text { - Largeur: } 1,9 \text { m min. en site contraint et } \\
2 \text { m min. en site non contraint, } \\
\text { bordure/marquage au sol compris. }\end{array}$ & $\begin{array}{l}\text { Définition: } \\
\text { Emprise destinée à la circulation des } \\
\text { deux-roues non motorisés sur trottoir ou en } \\
\text { site propre. La bande cyclable est une voie } \\
\text { contiguë à la chaussée, délimitée par des } \\
\text { bandes blanches peintes au sol. La piste } \\
\text { cyclable est séparée physiquement de la } \\
\text { chaussée utilisée par les véhicules à moteur. } \\
\text { Orientations d'aménagement: } \\
\text { - Élaboration d'un schéma directeur des } \\
\text { aménagements cyclables, desservant toutes } \\
\text { les communes. } \\
\text { - Aménagements sous forme de pistes, } \\
\text { marquages au sol ou couloirs indépendants, } \\
\text { en fonction des besoins et contraintes de la } \\
\text { circulation. } \\
\text { - Installation de délinéateurs (bornes, } \\
\text { balisettes) homogènes. } \\
\text { - Bande cyclable sur chaussée: } \\
\text { - } 1,2 \text { m au minimum et de manière } \\
\text { exceptionnelle, mais } 1,5 \text { à } 1,8 \text { m } \\
\text { souhaitable (unidirectionnelle), } \\
\text { marquage au sol et caniveau compris. } \\
\text { - de } 1,7 \text { à } 2 \text { m unidirectionnelle le long } \\
\text { du stationnement longitudinal, } \\
\text { marquage au sol et caniveau compris. } \\
\text { - Piste cyclable sur trottoir: } \\
\text { - Â intégrer sur les trottoirs de largeur } \\
\text { supérieure à } 3 \text { m en unidirectionnel et } \\
\text { (en site propre) : } \\
\text { - } 1,5 \text { m unidirectionnelles, } 2,5 \text { à } 3 \text { m } \\
\text { bidirectionnelles, entre obstacles. } \\
\text { - Les pistes cyclables ne pourront être } \\
\text { intégrées que sur les trottoirs de largeur } \\
\text { supérieure à } 3,5 \text { m. } \\
\text { Pistes cyclables sur chaussée } \\
\text { alroctionnel. }\end{array}$ & \\
\hline
\end{tabular}




\section{$2^{e}$ niveau d'agrégation}

Nous allons procéder par combinaison de deux ou trois indicateurs synthétiques, de façon à avoir un indice pour deux ou trois indicateurs synthétiques. Ce choix repose sur les cibles et les thématiques des différents critères (tableau 3). Nous les agrégeons selon la méthode $\operatorname{RST}_{02}{ }^{6}$ (Certu, 2006) pour avoir les valeurs d'indices. La valeur indicielle $\left(V I_{x^{\prime}}\right)$ est définie comme la moyenne des valeurs des indicateurs synthétiques.

$V I_{X^{\prime}}=\frac{1}{n^{\prime}} \sum_{i=1}^{x}$ VI. $S_{x}$

$n^{\prime} \quad=$ Le nombre d'indicateurs synthétiques combinés

VI. $S_{x}=$ La valeur des indicateurs synthétiques

Malgré l'existence d'un bon nombre d'approches plus raffinées et développées dans la littérature, nous avons retenu ces formes fonctionnelles qui se caractérisent par leur simplicité pour qu'elles soient facilement maniables ou intégrables par les décideurs publics.

Tableau 3 Tableau des indices, descripteurs des critères

\begin{tabular}{|c|c|}
\hline Critères & Indices \\
\hline Économie d'usage & \multirow{2}{*}{$\begin{array}{l}\text { Indice de durabilité } \\
\text { (ID) }\end{array}$} \\
\hline Gestion & \\
\hline Attractivité & \multirow{2}{*}{$\begin{array}{l}\text { Indice de performance fonctionnelle } \\
\qquad \text { (IPF) }\end{array}$} \\
\hline Sécurité et sûreté & \\
\hline Mobilité & \multirow{2}{*}{$\begin{array}{c}\text { Indice d'intégration fonctionnelle } \\
\text { (IIF) }\end{array}$} \\
\hline Accessibilité & \\
\hline Ambiances urbaines & \multirow{2}{*}{$\begin{array}{l}\text { Indice de qualité de vie } \\
\text { (IQV) }\end{array}$} \\
\hline Qualité environnementale & \\
\hline Composition urbaine & \multirow{2}{*}{$\begin{array}{l}\text { Indice de qualité du cadre de vie } \\
\text { (IQCV) }\end{array}$} \\
\hline Aménagement & \\
\hline
\end{tabular}

Nous synthétisons la procédure d'évaluation de la qualité des espaces publics dans le tableau 4 et, à titre d'exemple, nous présentons la méthode relative à 10 critères et 4 indicateurs/critère (figure 4).

6 La grille $\mathrm{RST}_{02}$ a été réalisée en 2006 par le Réseau scientifique et technique (RST) associant plusieurs organismes français (la Direction générale de l'urbanisme, de l'habitat et de la construction, les Centres d'études techniques de l'équipement [Cete] et le Centre d'études sur les réseaux, les transports, l'urbanisme et les constructions publiques [Certu]). Elle se veut un outil de questionnement et d'analyse, destiné à mettre en lumière les points faibles et les points forts d'un projet en regard de critères du développement durable. 


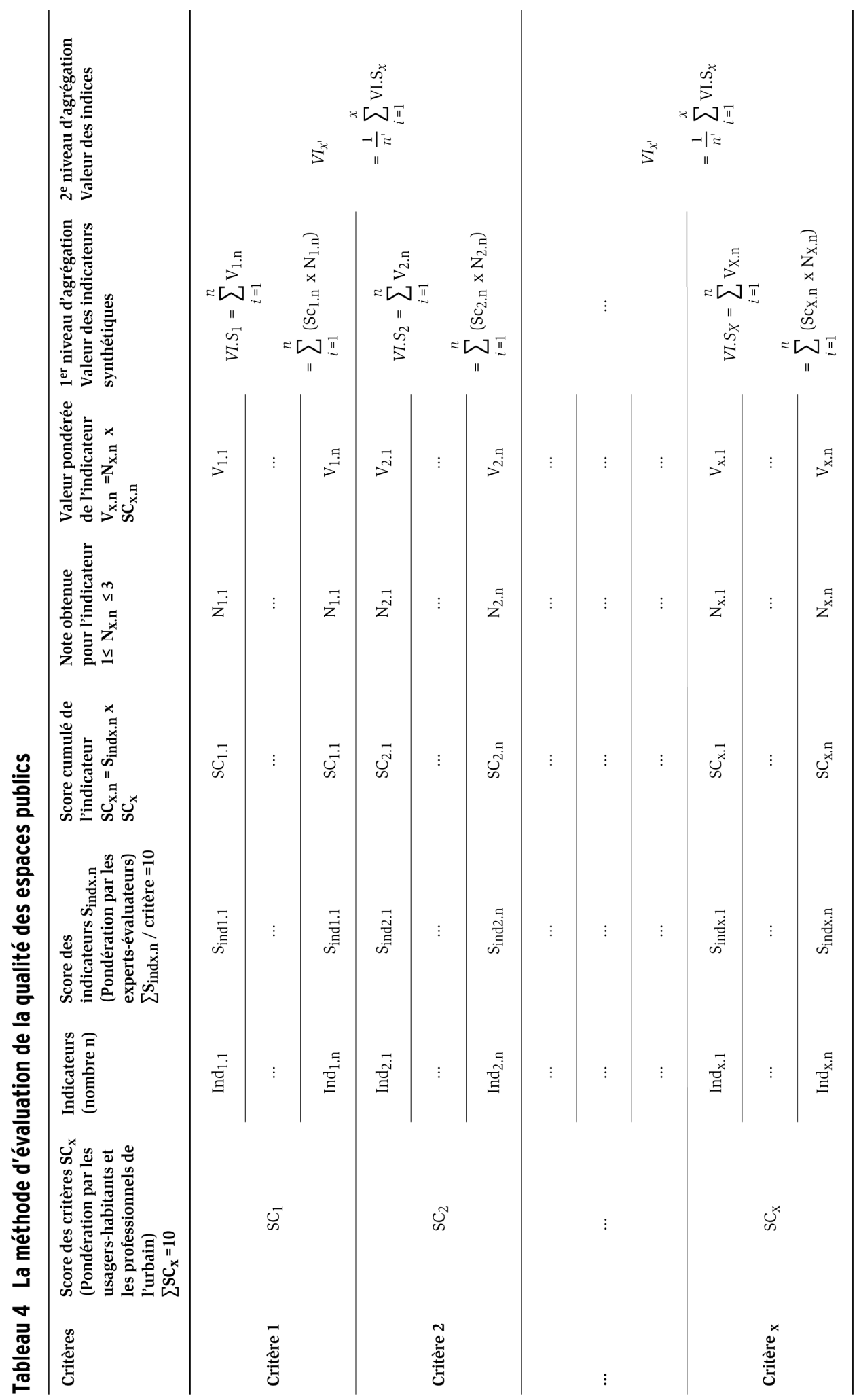


Figure 4 Procédure d'évaluation de la qualité des espaces publics

Méthode QUEP
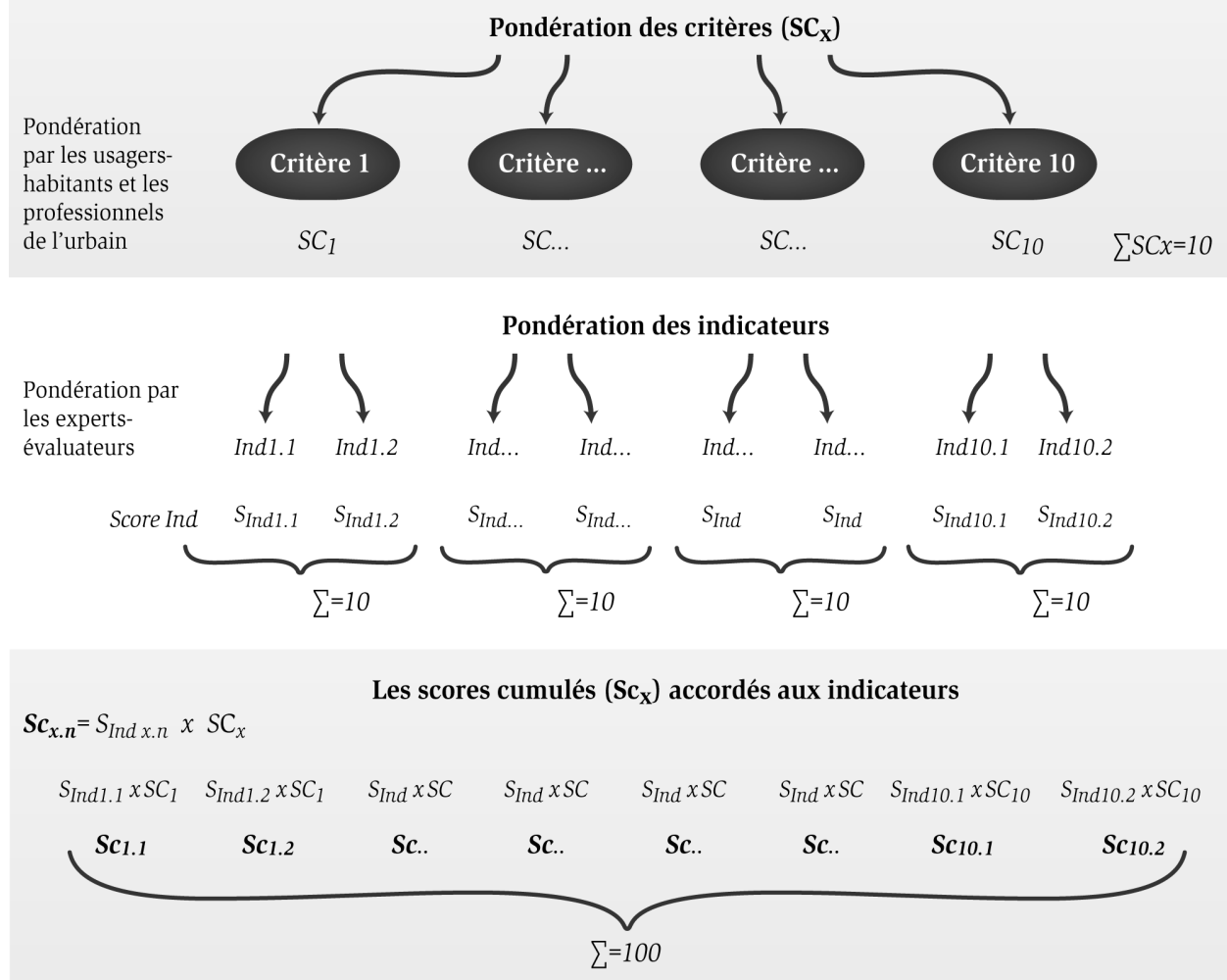

Attribution des notes aux indicateurs $\mathrm{N}_{\mathrm{X} . \mathrm{n}}$
$N_{1.1}$
$N_{1.2}$
$N$
N..
$N$.
$N$
$N_{10.1}$
$N_{10.2}$

Valeur pondérée de l'indicateur $\left(\mathrm{V}_{\mathrm{X} . \mathrm{n}}\right)$

$\begin{array}{cccccccc}\boldsymbol{S c}_{1.1} \times N_{1.1} & \mathbf{S c}_{1.2} \times N_{1.2} & \boldsymbol{S c} \times N & \boldsymbol{S c} \times N & \boldsymbol{S c} \times N & \boldsymbol{S c} \times N & \boldsymbol{S c}_{10.1} \times N_{10.1} & \boldsymbol{S c}_{10.2} \times N_{10.2} \\ V_{1.1} & V_{1.2} & V . . & V . . & V . . & V_{. .} & V_{10.1} & V_{10.2}\end{array}$

Agrégation $1^{\text {er }}$ niveau (Méthode WSM)

Valeurs des indicateurs synthétiques (VI.S)

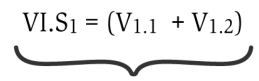

VI.S 1

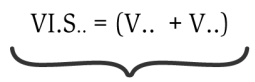

VI.S..

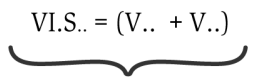

VI.S.

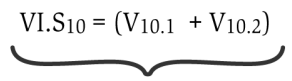

VI.S 10

\section{Agrégation $2^{\mathrm{e}}$ niveau (Méthode $\mathrm{RST}_{\mathbf{0 2}}$ )} Valeurs des indices (VI)

$$
V_{1}=(\text { VI.S } 1+\text { VI.S }) / 2 \quad V I_{2}=\left(V_{1} . S_{3}+\text { VI.S }\right) / 2 \quad \text { VI.. }=(\text { VI.S.. }+ \text { VI.S.. }) / 2 \quad V I 5=\left(V I . S_{9}+\text { VI.S } 10\right) / 2
$$




\section{Faisabilité opérationnelle de la démarche: cas des espaces publics aux abords de la station de métro 1er mai}

Le métro d'Alger est un projet qui date des années 1970 pour parer à l'explosion démographique et au besoin de transport collectif qui en résulte. Il a été lancé dans les années 1980 et arrêté dans les années 1990 à cause des problèmes financiers et de l'insécurité, puis relancé en 2003.

La ligne 1 Hai-El Badr-Tafourah-Grande poste est d'une longueur de 8,6 km et comporte 10 stations dont la station $1^{\mathrm{er}}$ mai (figures 5 et 6 ).

Il y a eu une amélioration des déplacements et un changement d'usage en termes de choix de mobilité, par la mise en service de la ligne 1 du métro. Un changement de pratique implique un renouvellement certain des espaces publics se trouvant aux abords des stations de métro, ce qui n'est pas totalement le cas à Alger, sauf dans quelques pôles d'échanges.

Figure 5 Plan de situation de la station $1^{\text {er }}$ mai

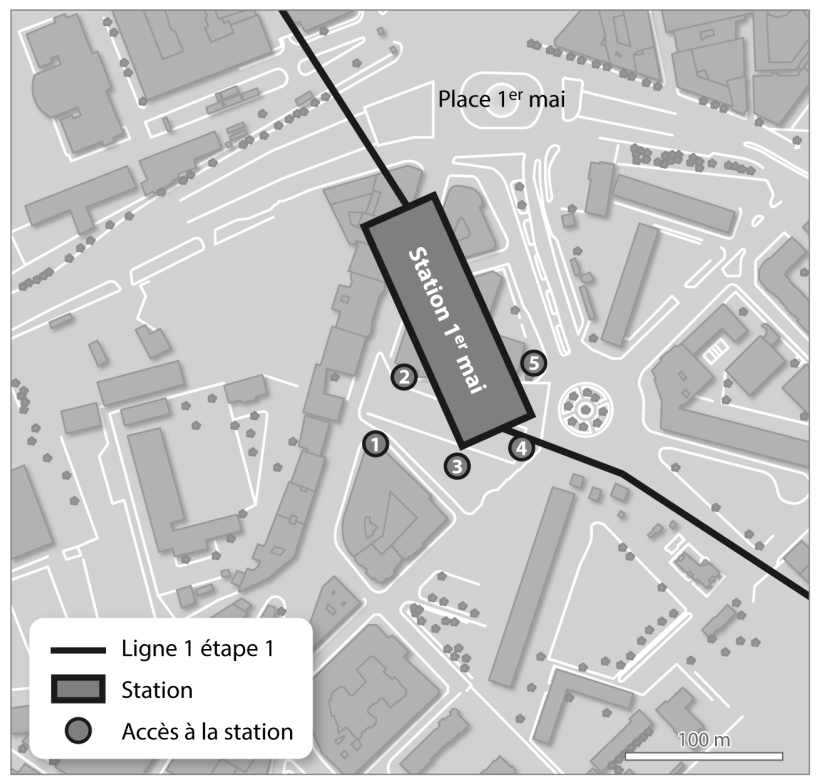

Source: Entreprise Métro d'Alger

Nous avons testé la faisabilité opérationnelle de la démarche à travers une évaluation ex-post sur le cas de la station $1^{\mathrm{er}}$ mai, en raison de sa position stratégique au cœur de l'hyper-centre d'Alger. Après les étapes de pondération, de notation et d'agrégation (tableau 5), nous avons comparé la valeur indicielle VI(figure 7) avec la valeur maximale possible de chaque indice $V I_{M A X}$ (équation 2) et un «seuil de performance» défini comme le $2 / 3$ de cette valeur maximale. Nous considérons que la performance d'un projet sur le plan de la qualité de ses espaces publics ne peut dépasser les $2 / 3$ de la valeur maximale, et cela, pour plusieurs raisons : le contexte, les moyens mis à disposition pour la réalisation du projet, les contraintes techniques, les coûts, etc.). 
Figure 6 Abords de la station $1^{\text {er }}$ mai
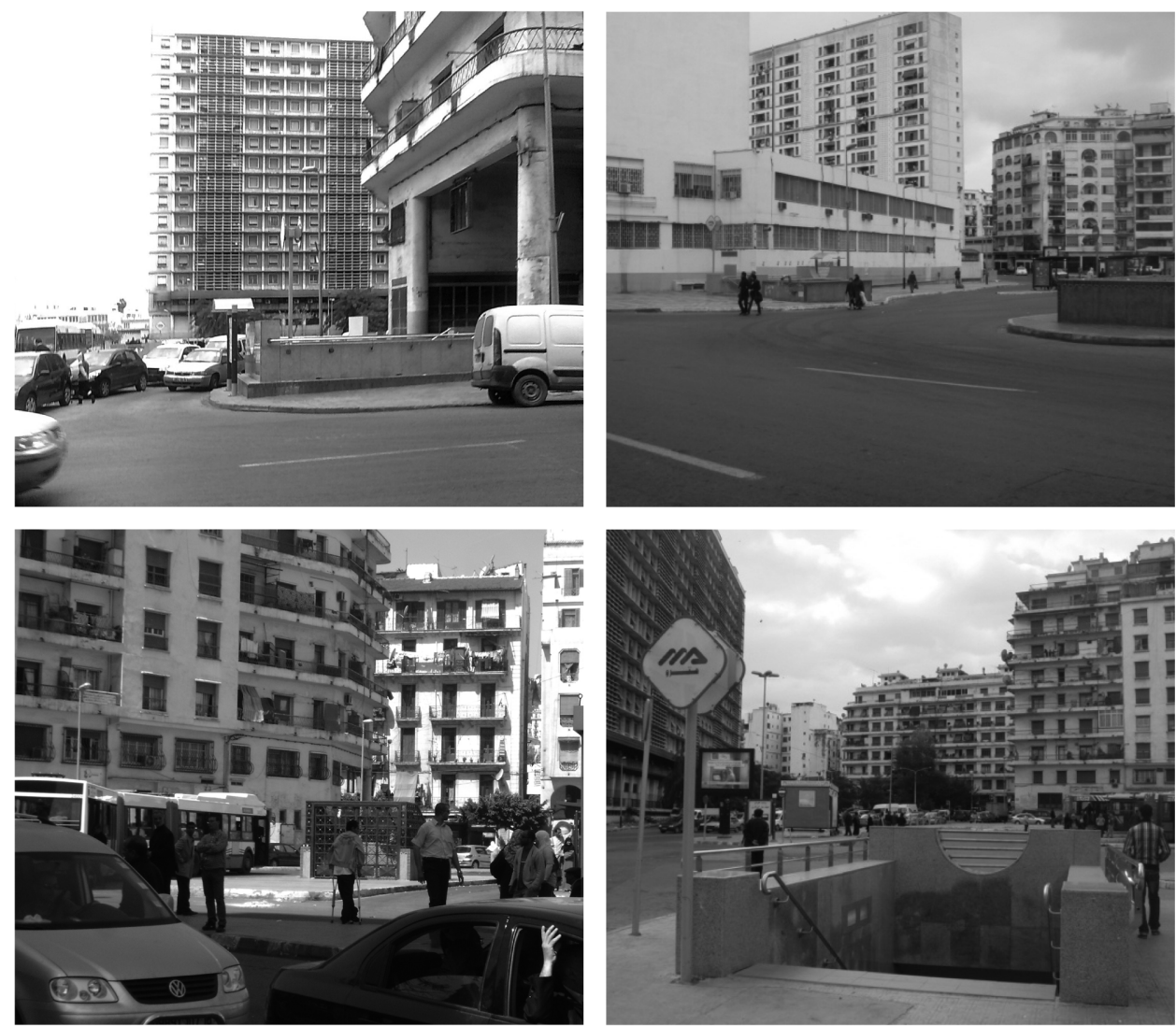

Source: HADJI. Lydia, 2012

Pour un arbre de pondération à 10 critères et 4 indicateurs/critère ${ }^{7}$

Score maximal attribué au critère: $1 \quad(\Sigma$ Scores $=10)$

Score maximal indicateur: $7 \quad ;(\Sigma$ Score indicateurs $/$ critère $=10)$

Score maximal cumulé: $7 \times 1=7 \quad$; note maximale: 3

Valeur maximale pondérée de l'indicateur $\mathrm{V}_{\text {imax }}: 7 \times 1 \times 3=21$

Valeur maximale de l'indicateur synthétique VI.S ${ }_{\max }:(7 \times 1 \times 3+1 \times 1 \times 3+1 \times 1 \times 3+1 \times 1 \times 3)=$ $(21+3+3+3)=30$.

Valeur maximale de l'indice $V I_{M A X}=\left(V_{I} . S_{1}+V I . S_{2}\right) / 2=(30+30) / 2=60 / 2=30$

Seuil de performance de l'indice $=30 \times 2 / 3=20$

7 Pour l'application de la méthode sur le cas d'étude algérois, nous avons réduit le nombre d'indicateurs à 40 (4 indicateurs par critère). 
Tableau 5 Extrait du tableau des valeurs des indices de qualité des espaces publics

\begin{tabular}{|c|c|c|c|c|}
\hline $\begin{array}{l}\text { Critères } \\
\text { synthétiques } \\
\text { d'évaluation }\end{array}$ & $\begin{array}{l}\text { Indicateurs d'évaluation de } \\
\text { la qualité de l'espace public }\end{array}$ & $\begin{array}{l}\text { Valeurs } \\
\text { pondérées des } \\
\text { indicateurs }\end{array}$ & $\begin{array}{l}\text { Valeurs des } \\
\text { indicateurs } \\
\text { synthétiques }\end{array}$ & $\begin{array}{l}\text { Valeur } \\
\text { de } \\
\text { l'indice }\end{array}$ \\
\hline \multirow{4}{*}{ Mobilité } & $\begin{array}{l}\text { Offre de places de stationnement aux abords } \\
\text { des stations }\end{array}$ & 3,15 & \multirow{4}{*}{23,1} & \multirow{8}{*}{23,1} \\
\hline & $\begin{array}{l}\text { Offre d'espaces « deux-roues » (modes doux) } \\
\text { aux abords des stations }\end{array}$ & 1,05 & & \\
\hline & $\begin{array}{l}\text { Offre d'espaces d'accueil de flux piétons aux } \\
\text { abords des stations (parvis) }\end{array}$ & 12,60 & & \\
\hline & Largeur des voies pour les espaces véhicules & 6,30 & & \\
\hline \multirow{4}{*}{ Accessibilité } & $\begin{array}{l}\text { Existence de cheminements adaptés et sans } \\
\text { obstacles }\end{array}$ & 9,90 & \multirow{4}{*}{23,1} & \\
\hline & $\begin{array}{l}\text { Existence d'aménagements spécifiques pour } \\
\text { les personnes à mobilité réduite (PMR) }\end{array}$ & 6,60 & & \\
\hline & $\begin{array}{l}\text { Existence de panneaux de signalisation et } \\
\text { des éléments de repère }\end{array}$ & 3,30 & & \\
\hline & $\begin{array}{l}\text { Offre de places de stationnement } \\
\text { temporaire (approvisionnement, urgence...) }\end{array}$ & 3,30 & & \\
\hline
\end{tabular}

\section{Figure 7 Performance de la qualité des espaces publics aux abords de la station $1^{\text {er }}$ mai}

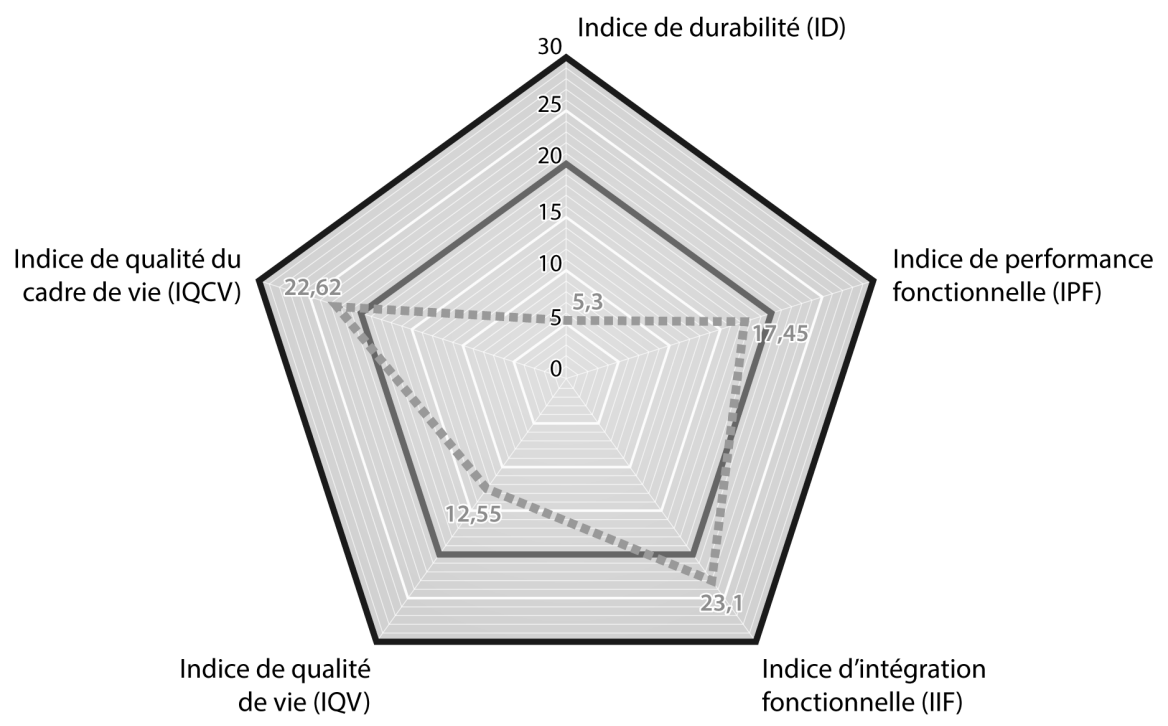


Les indices de durabilité (ID), de qualité de vie (IQV) et de performance fonctionnelle (IPF), dont les valeurs sont au-dessous du seuil de performance, sont considérés comme non performants. Ils marquent les défaillances du projet qui ne sont pas du tout acceptables pour son avenir. Cela démontre l'indifférence des acteurs urbains (maître d'ouvrage et maître d'œuvre) à l'égard des principes du développement durable. Ces défaillances sont dues surtout à l'absence d'un cahier des charges dont l'importance se résume dans l'étude et dans le processus d'aide à la décision. Ce règlement doit être accompagné d'une charte des espaces publics.

Les valeurs d'indices d'intégration fonctionnelle (IIF) et de qualité du cadre de vie (IQCV) dépassant le seuil de performance s'expliquent par la position stratégique dans laquelle se trouve la station de métro $1^{\text {er }}$ mai et la vocation de ce projet, qui est celle de développer la mobilité douce.

\section{Conclusion et perspectives d'application de la méthode d'évaluation de la qualité des espaces publics}

Cet outil d'aide à la décision est simple d'utilisation, compréhensible et pourrait s'appliquer à différents niveaux d'un projet d'espace public: ex-ante, in itinéraire (intermédiaire) et ex-post. Son application conduit à apporter des modifications, formuler des recommandations et élaborer ou éventuellement améliorer les cahiers des charges.

La méthode est holistique et participative, car elle permet de n'oublier aucun aspect et rassemble plusieurs acteurs urbains, y compris les usagers. Elle réduit aussi le temps de réflexion des décideurs en ciblant les indicateurs défaillants sur lesquels il faut agir.

Cette méthode a cependant des limites, compte tenu du degré de subjectivité que comprend le système de pondération. La quantité et la précision des informations disponibles influent sur le mode de calcul.

Cette approche holistique de l'espace public n'est qu'une partie des problématiques de l'éventail systémique qui se base sur les cibles du développement durable et dont la complexité pourrait constituer l'objet d'autres recherches en vue d'identifier le poids réel des critères d'évaluation. Notre objectif majeur est de transformer la méthode en logiciel, ce qui pourrait faciliter son usage et élargir le champ d’application. 


\section{Bibliographie}

BANA E COSTA Carlos. A, DE CORTE JeanMarie et VANSNICK Jean-Claude (2005) On the mathematical foundation of MACBETH. Multiple Criteria Decision Analysis: State of the Art Surveys. New York, Springer, p. 409-437.

BEREZOWSKA-AZZAG, Ewa (2012) Projet urbain: comprendre la démarche du projet urbain. Alger, Éditions Synergie.

BOULANGER, Paul-Marie (2004) Les indicateurs de développement durable: un défi scientifique, un enjeu démocratique. Les séminaires de l'Iddri, no 12 .

CERTU (2006) Prendre en compte le développement durable dans un projet - Guide d'utilisation de la grille RST ${ }_{02}$ Lyon, CERTU.

CHARLOT-VALDIEU, Catherine et OUTREQUIN, Philippe (2009) L'urbanisme durable-Concevoir un éco quartier. Paris, Éditions Le Moniteur.

CHERQUI, Frédéric (2005) Méthodologie d'évaluation d'un projet d'aménagement durable d'un quartier - Méthode ADEQUA. La Rochelle, Université La Rochelle, thèse de doctorat en génie civil.

CHURCHMAN C. West et ACKOFF, Russell L. (1954) An approximate measure of value. Journal of the Operational Research Society of America, vol. 2, no 2, p. 172-187.

CULLEN, Gordon (1961) The concise townscape. London, Architectural Press.

DEVILLARD, Valérie et JANNIÈRE, Hélène (1997) Espace public - communauté et voisinage 1945-1955. Dans Virginie Picon-Lefebvre (dir.) Les espaces publics modernes - Situations et propositions. Paris, Le Moniteur.

GOFFMAN, Erwin (1973) La mise en scène de la vie quotidienne. Les relations en public, vol.2. Paris, Les éditions de Minuit.
HADJI, Lydia (en cours) Les espaces publics face aux stratégies du développement $d u-$ rable urbain-Méthode d'évaluation de la qualité des espaces publics aux abords des stations de métro et de tramway à Alger. Thèse de doctorat en sciences, (option Architecture et Urbanisme). Alger, École Polytechnique d'Architecture et d'Urbanisme (EPAU).

HALL, Edward T. (1966) La dimension cachée. Paris, Éditions du Seuil.

LYNCH, Kevin (1969) [1960] L'image de la cité. Paris, Dunod.

ROY, Bernard et BOUYSSOU, Denis (1993) Aide multicritère à la décision: méthodes et cas. Paris, Economica.

SAATY, Thomas L. (1977) A scaling method for priorities in hierarchical structures. Journal of Mathematical Psychology, vol. 15, p. 231-281.

SENETT, Richard (1979) Les tyrannies de l'intimité. Paris, Éditions du Seuil.

SEN, Amartya (1993) Capability and wellbeing. Dans Martha Nussbaum et Amartya Sén (dir.) The quality of life. Oxford, Clarendon Press. 